\title{
Energy uniform decay rates for the semilinear wave equation with nonlinear localized damping and source terms of critical variable exponent
}

\author{
Abita Rahmoune \\ Laboratory of Pure and Applied Mathematics, University of Laghouat, P.O. Box 37G, Laghouat, Algeria
}

Received: 13 June 2021, Accepted: 16 June 2021

Published online: 4 July 2021.

\begin{abstract}
The goal of this paper is to consider a model of a semilinear wave equation with critical source term $f(u(x, t))$ and a localized exterior nonlinear monotone damping $g\left(u_{t}(x, t)\right)$. The proposed approach allows considering, in a unified way, much more general classes of hyperbolic problems than addressed before in the literature. This generalization refers to an analysis aspect of the problem. The method leads to optimal decay rates for solutions of semilinear hyperbolic equations driven by a source of critical variable exponent and subjected to nonlinear damping localized in a small region adjacent to a portion of the boundary. The no-growth condition is imposed on the damping $g\left(u_{t}(x, t)\right)$, and this is the distinct feature of the model include. Hence we consider a more general case of $g$, and the sole conditions assumed are, $g$ has not a polynomial behavior near the origin, monotonicity, continuity, $g(0)=0$. In particular, no differentiability and no growth conditions are imposed on the damping both at the origin and at the infinity. The asymptotic decay rates for the energy function obtained by using Martinez's method [11], however, this paper can be regarded as a generalization of Martinez's results.
\end{abstract}

Keywords: Wave equation, localized damping, source, Decay rates, critical exponents, variable nonlinearity.

\section{Introduction}

In this work, we are concerned with the uniform decay rates of solutions of the semilinear wave equation with variableexponent nonlinearities and nontrivial boundary conditions

$$
\begin{gathered}
u_{t t}-\Delta u+f(u)=0 \text { in } \Omega \times(0,+\infty), \\
u=0 \text { on } \Gamma_{0} \times(0,+\infty), \\
\frac{\partial u}{\partial v}+g\left(u_{t}(x, t)\right)=|u|^{p(x)-2} u \text { on } \Gamma_{1} \times(0,+\infty), \\
u(x, 0)=u_{0}(x), u_{t}(x, 0)=u_{1}(x), x \in \Omega,
\end{gathered}
$$

where $\Omega \subset \mathbb{R}^{n}(n \geq 2)$, be a bounded domain with a smooth boundary $\Gamma=\partial \Omega=\Gamma_{0} \cup \Gamma_{1}$. The boundary $\Gamma$ of $\Omega$ is assumed to be regular and is divided by two closed and disjoint parts $\Gamma_{0}, \Gamma_{1}$, Here, $\Gamma_{0} \neq \emptyset$. (.) denotes the derivative with respect to time $t$ thus $u_{t}=\frac{\partial u}{\partial t}, u_{t t}=\frac{\partial^{2} u}{\partial t^{2}}, \Delta$ stands for the Laplacian with respect to the spatial variables. Let $v$ be the outward normal to $\Gamma$. The exponent $p($.$) is given measurable function on \Omega$

Constant exponent: When $p$ is constant, for problem (1)-(4), many results have been obtained, such as the existence and uniqueness in [2], [16], [17], [18], [26], [12], [14], [15], [24], [21] and a list of references therein, blow-up in finite time in [9], [8], energy decay estimate in [23], [11], boundary stabilization in [13], [1], [25]. 
In recent years, much attention has been paid to the study of mathematical nonlinear models of hyperbolic, parabolic and elliptic equations with nontrivial boundary conditions. In fact, there are only a few works regarding problems with nontrivial boundary conditions. Let us mention some of these problems.

For instance, Vitillaro [22] considered the wave equation with linear interior-boundary damping terms and nonlinear boundary source terms. He proved global nonexistence for the problem using the convexity method but did not prove blow-up results. Cavalcanti et al. [2] studied blow-up for the linear wave equation with boundary damping and interior-boundary source terms under suitable conditions.

Recently, Tae Gab Ha et al. in [25], [16], [7], [19] a semi-linear wave equations associated with the Laplace operator, polynomial nonlinear, boundary damping and source term has been considered, they have proved blow-up of solutions with positive initial energy by using potential well theory and contradiction method, they study energy decay using the multiplier technique and equivalent energy, they show energy decay rates for solutions and prove a finite time blow-up result under suitable conditions on the initial data and positive initial energy.

The author in [20] generalized some of the previous results so that $g\left(u_{t}\right)=\left|u_{t}\right|^{m(.)-2} u_{t}$ and the exponent $p$ and $m$ in the boundary conditions are measurable functions. By perturbing the boundary equation, he proved the existence of solutions and uniform decay rates of the wave equation with boundary damping and source term.

The goal of this paper is to study the uniform decay rate of the energy to the wave equation with variable-exponent nonlinearities and nontrivial boundary conditions. To our knowledge, in this case, there is no uniform decay rates of solutions of the wave equation with boundary damping and source terms as in (1)-(4), then we may be able to analyze the questions of the asymptotic stability of solutions.

In this work, we consider (1)-(4). We intend to prove the uniform decay rates of the wave equation with boundary damping and source term involving variable exponents.

This paper consists of 3 sections in addition to the introduction. In Section 2, we recall the definitions of the variable-exponent Lebesgue spaces $L^{p(.)}(\Omega)$, the Sobolev spaces $W^{1, p(.)}(\Omega)$, and some of their properties, and we present, without proof, a well-posedness result for the problem (1)-(4). In Section 3, we state, with the proof, the stability theorem for certain solutions with positive initial energy. To the best of our knowledge, this problem has not been studied previously. Besides, our method of determining these results, because the presence of the exponent $p($.$) , is somewhat$ different from step to step.

\section{Preliminaries: Function spaces}

In this section, we list and recall some well-known results and facts from the theory of the Sobolev spaces with variable exponent. (On the basic properties of the spaces $W^{1, p(x)}(\Omega)$ and $L^{p(x)}(\Gamma)$ we refer to [3], [4], [5], [6], [10]).

Throughout the rest of the paper we assume that $\Omega$ is a bounded domain of $\mathbb{R}^{n}, n \geq 1$ with smooth boundary $\Gamma$ and assume that $p($.$) is a measurable function on \bar{\Omega}$ such that:

$$
2<p^{-} \leq p(x) \leq p^{+} \leq p_{*}(x)<\infty,
$$


where

$$
p^{+}=\underset{x \in \Omega}{e s s} \sup p(x), p^{-}=\underset{x \in \Omega}{e s s} \inf p(x) .
$$

and

$$
p_{*}(x)=\left\{\begin{array}{l}
\frac{n p(x)}{(n-p(x))^{+}} \text {if } n>p^{+} \\
+\infty \text { if } n \leq p^{+} .
\end{array}\right.
$$

We also assume that $p$ satisfies the following Zhikov-Fan uniform local continuity condition :

$$
|p(x)-p(y)| \leq \frac{M}{|\log | x-y||} \text {, for all } x, y \text { in } \Omega \text { with }|x-y|<\frac{1}{2}, M>0 .
$$

Given a function $p: \bar{\Omega} \rightarrow\left[p^{-}, p^{+}\right] \subset(1, \infty), p^{ \pm}=$const, we define the set

$$
L^{p(.)}(\Omega)=\left\{\begin{array}{c}
v: \Omega \rightarrow \mathbb{R}: v \text { measurable functions on } \Omega, \\
\rho_{p(.), \Omega}(v)=\int_{\Omega}|v(x)|^{p(x)} \mathrm{d} x<\infty .
\end{array}\right\}
$$

The variable-exponent space $L^{p(\cdot)}(\Omega)$ equipped with the Luxemburg norm

$$
\|u\|_{p(.)}=\|u\|_{L^{p(.)}(\Omega)}=\inf \left\{\lambda>0, \int_{\Omega}\left|\frac{u}{\lambda}\right|^{p(x)} \mathrm{d} x \leq 1\right\}
$$

becomes a Banach space.

In general, variable-exponent Lebesgue spaces are similar to classical Lebesgue spaces in many aspects, see the first discussed the $L^{p(x)}$ spaces and $W^{k, p(x)}$ spaces by Kovacik and Rakosnik in [10].

Let us list some properties of the spaces $L^{p(\cdot)}(\Omega)$ which will be used in the study of the problem (1)-(4).

If $p(x)$ is measurable and $1<p^{-} \leq p(x) \leq p^{+}<\infty$ in $\Omega$, then $L^{p(\cdot)}(\Omega)$ is a reflexive and separable Banach space and $C_{0}^{\infty}(\Omega)$ is dense in $L^{p(\cdot)}(\Omega)$.

It follows directly from the definition of the norm that

$$
\min \left(\|u\|_{p(.)}^{p^{-}},\|u\|_{p(.)}^{p^{+}}\right) \leq \rho_{p(.)}(u) \leq \max \left(\|u\|_{p(.)}^{p^{-}},\|u\|_{p(.)}^{p^{+}}\right) .
$$

\subsection{Mathematical hypotheses}

We begin this subsection by introducing some hypotheses and our main result. Throughout this paper,we use standard functional spaces and denote that (.,.), \|. \| the inner products and norms in $L^{2}(\Omega)$ and $H_{0}^{1}(\Omega)$ represented and they are given by:

$$
\begin{gathered}
(u, v)=\int_{\Omega} u(x) v(x) \mathrm{d} x \text { and }\|u\|_{L^{2}(\Omega)}^{2}=\int_{\Omega} u^{2} \mathrm{~d} x ; \\
\|u\|_{H_{0}^{1}(\Omega)}^{2}=\|u\|^{2}=\int_{\Omega}|\nabla u|^{2} \mathrm{~d} x,
\end{gathered}
$$


And $\|\cdot\|_{p(.)},\|\cdot\|_{p(.), \Gamma_{1}}$ are $L^{p(.)}(\Omega)$ norm and $L^{p(.)}\left(\Gamma_{1}\right)$ norm, respectively, such that:

$$
\|u\|_{p(.), \Gamma_{1}}=\int_{\Gamma_{1}}|u(\eta)|^{p(\eta)} \mathrm{d} \eta=\int_{\Gamma_{1}}|u(x)|^{p(x)} \mathrm{d} \Gamma
$$

Also, we define $(u, v)_{\Gamma_{1}}=\int_{\Gamma_{1}} u(x) v(x) \mathrm{d} \Gamma$.

(H1) Hypotheses on $\Omega$ (Basic geometrical condition):Let $\Omega \subset \mathbb{R}^{n}(n \geq 2)$, be a bounded domain with a smooth boundary $\Gamma=\partial \Omega$. The boundary $\Gamma$ of $\Omega$ is assumed to be regular and is divided by two closed and disjoint parts $\Gamma_{0}$, $\Gamma_{1}$, with $\Gamma_{0} \neq \emptyset$, satisfying the following conditions

$$
\begin{aligned}
s(x) \cdot v(x) & \geq \sigma>0 \text { on } \Gamma_{1}, \quad s(x) \cdot v(x) \leq 0 \text { on } \Gamma_{0}, \\
s(x) & =x-x^{0}\left(x^{0} \in \mathbb{R}^{n}\right) \text { and } R=\max _{x \in \bar{\Omega}}|s(x)|,
\end{aligned}
$$

where $v$ denote the unit outward normal to $\Gamma$ and $x^{0}$ be a fixed point in $\mathbb{R}^{n}$. And we assume that:

$$
\frac{\partial u_{0}}{\partial v}+g\left(u_{1}\right)=\left|u_{0}\right|^{p(x)-2} u_{0} \text { on } \Gamma_{1}
$$

(H2) Hypotheses on $f:$ Let $f: \mathbb{R} \rightarrow \mathbb{R}$ is a Lipschitz function and satisfy:

$$
f(s) s \geq 0 \text { for all } s \in \mathbb{R}
$$

and there exists a positive constant $\delta$ such that:

$$
(n-1) f(s) s \geq(2 n+\delta) F(s),
$$

where $F(s)=\int_{0}^{s} f(r) \mathrm{d} r$.

(H3) Hypotheses on $g:$ Let $g: \mathbb{R} \rightarrow \mathbb{R}$ be a nondecreasing $C^{1}$ such that $g(0)=0$ and suppose that there exists a strictly increasing and odd function $\beta$ of $C^{1}$ class on $[-r, r](r \geq 1)$ such that

$$
\begin{gathered}
|\beta(s)| \leq|g(s)| \leq \beta^{-1}(s) \text { if }|s| \leq r, \\
c_{1}|s| \leq|g(s)| \leq c_{2}|s| \text { if }|s|>r .
\end{gathered}
$$

To simplify the situation we take $r=1$, where $\beta^{-1}$ denotes the inverse function of $\beta$ and $c_{1}, c_{2}$ are positive constants. (H4) Hypotheses on $p($.$) :Let p($.$) is given measurable functions on \bar{\Omega}$ satisfying the following condition,

$$
\begin{gathered}
2<p^{-} \leq p(x) \leq p^{+}<\frac{2 n-3}{n-2} \text { if } n \geq 3 \\
\text { and } p^{+} \geq 2 \text { if } n=1,2
\end{gathered}
$$

Furthermore let $B>0$ be the optimal constant of Sobolev embedding $H_{\Gamma_{1}}^{1}(\Omega) \hookrightarrow L^{p(.)}\left(\Gamma_{1}\right)$ which satisfies the inequality

$$
\|u\|_{p(.), \Gamma_{1}} \leq B\|\nabla u\|_{2} \quad \forall u \in H_{\Gamma_{1}}^{1}(\Omega) .
$$

where

$$
H_{\Gamma_{1}}^{1}(\Omega)=\left\{u \in H^{1}(\Omega):\left.u\right|_{\Gamma_{1}}=0\right\}
$$


endow with the Hilbert structure induced by $H^{1}(\Omega)$, is a Hilbert space. Let us consider

$$
J(u)=\frac{1}{2}\|\nabla u\|_{2}^{2}-\int_{\Gamma_{1}} \frac{1}{p(x)}|u|^{p(x)} \mathrm{d} \Gamma, u \in H_{\Gamma_{0}}^{1}(\Omega) .
$$

Moreover, suppose that

$$
E(0)<\mathrm{d} \text { and }\left\|\nabla u_{0}\right\|_{2}^{2}=\alpha_{0}<\alpha_{1} \leq B_{1}^{-2},
$$

where $\mathrm{d}$ define by the positive number

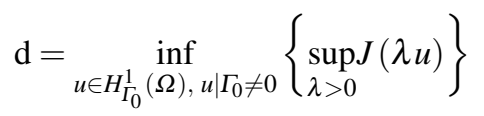

where $C_{f}$ is a Lipschitz constant. In order to formulate our results, it is convenient to introduce the energy associated with problem (1)-(4):

$$
\begin{gathered}
E(t)=\frac{1}{2}\left\|u_{t}\right\|_{2}^{2}+\frac{1}{2}\|\nabla u\|_{2}^{2}+\int_{\Omega} F(u(x, t)) \mathrm{d} x-\int_{\Gamma_{1}} \frac{1}{p(x)}|u|^{p(x)} \mathrm{d} \Gamma \\
=\frac{1}{2}\left\|u_{t}\right\|_{2}^{2}+\int_{\Omega} F(u(x, t)) \mathrm{d} x+J(u),
\end{gathered}
$$

then

$$
E^{\prime}(t)=-\int_{\Gamma_{1}} g\left(u_{t}\right) \mathrm{d} \Gamma
$$

So the energy $E(t)$ is a nonincreasing function.

Furthermore, we have

$$
0<\mathrm{d}=h\left(\alpha_{1}\right)=\left(\frac{1}{2}-\frac{1}{p^{-}}\right) \alpha_{1}
$$

Now, if one considers

$$
\|\nabla u\|_{2}^{2}<\alpha_{1} \leq B_{1}^{-2}
$$

by (7) and (14)-(18), we have for any $t \geq 0$

$$
\begin{aligned}
E(t) & \geq J(u(t)) \geq \frac{1}{2}\|\nabla u\|_{2}^{2}-\frac{1}{p^{-}} \int_{\Gamma_{1}}|u|^{p(x)} \mathrm{d} \Gamma_{1} \geq \frac{1}{2}\|\nabla u\|_{2}^{2}-\frac{1}{p^{-}} \max \left(\|u\|_{p(.), \Gamma_{1}}^{p^{+}}\|u\|_{p^{(.)}, \Gamma_{1}}^{p^{-}}\right) \\
& \geq \frac{1}{2}\|\nabla u\|_{2}^{2}-\frac{1}{p^{-}} \max \left(B_{1}^{p^{+}}\|\nabla u\|_{2}^{p^{+}}, B_{1}^{p^{-}}\|\nabla u\|_{2}^{p^{-}}\right)=h\left(\|\nabla u\|_{2}^{2}\right)=\left(\frac{1}{2}-\frac{1}{p^{-}}\|\nabla u\|_{2}^{p^{-}-2} B_{1}^{p^{-}}\right)\|\nabla u\|_{2}^{2} \\
& \geq\left(\frac{1}{2}-\frac{1}{p^{-}} \alpha_{1}^{\frac{p^{-}-2}{2}} B_{1}^{p^{-}}\right)\|\nabla u\|_{2}^{2}=\left(\frac{1}{2}-\frac{1}{p^{-}}\right)\|\nabla u\|_{2}^{2}=\frac{p^{-}-2}{2 p^{-}}\|\nabla u\|_{2}^{2}, u \in H_{\Gamma_{0}}^{1}(\Omega)
\end{aligned}
$$

and, consequently,

$$
J(u(t)) \geq 0 \text { and } J(u(t))=0 \text { iff } u=0
$$

and

$$
\|\nabla u\|_{2}^{2} \leq \frac{2 p^{-}}{\left(p^{-}-2\right)} E(t) \leq \frac{2 p^{-}}{\left(p^{-}-2\right)} E(0) .
$$

\subsection{Existence of local solutions}

This subsection first present, without proof, a well-posedness result for the problem (1)-(4) with a degenerated second order equation on $\Gamma_{1}$. By perturbing the boundary equation, based on the combination of the Faedo-Galerkin 
approximation and the compactness method together with the Banach fixed point theorem, we can establish the proof, by combining the arguments as in [20], [25].

Theorem 1. Suppose that

$$
\left(u_{0}, u_{1}\right) \in\left\{\begin{array}{c}
H_{\Gamma_{0}}^{1}(\Omega) \cap H^{2}(\Omega) \times H_{\Gamma_{0}}^{1}(\Omega) \\
E(0)<\mathrm{d} \text { and }\left\|\nabla u_{0}\right\|_{2}^{2}=\alpha_{0}<\alpha_{1} \leq B_{1}^{-2}
\end{array}\right\} .
$$

Consider the above hypotheses. Then the problem (1)-(4)possesses a unique weak solution $u(t)$ satisfying:

$$
\begin{gathered}
u \in L^{\infty}\left(0, T ; H_{\Gamma_{0}}^{1}(\Omega) \cap H^{2}(\Omega)\right) \cap C^{1}\left(0, T ; L^{2}(\Omega)\right), \\
u_{t} \in L^{\infty}\left(0, T ; H_{\Gamma_{0}}^{1}(\Omega)\right) \\
u_{t t} \in L^{\infty}\left(0, T ; L^{2}(\Omega)\right) .
\end{gathered}
$$

Now we are in a position allowing us to state our result.

Theorem 2. Assume that hypotheses (H1)-(H4) hold. Then we have:

$$
E(t) \leq C_{3}\left(G^{-1}\left(\frac{1}{t}\right)\right)^{2}
$$

where $G(s):=s \beta(s)$ and $C_{3}$ is a positive constant that depends only on $E(0)$. Moreover, if the function $N(s):=\frac{\beta(s)}{s}$ is nondecreasing on $[0, \eta]$ for some $\eta>0$ and $N(0)=0$, then:

$$
E(t) \leq C_{4}\left(\beta^{-1}\left(\frac{1}{t}\right)\right)^{2}
$$

where $C_{4}$ is a positive constant that depends only on $E(0)$.

To end this subsection, we evoke a special lemma that will play a crucial role when establishing the asymptotic behavior.

Lemma 1. (See [11])Let $E: \mathbb{R}^{+} \rightarrow \mathbb{R}^{+}$be a non increasing function and $\phi: \mathbb{R}^{+} \rightarrow \mathbb{R}^{+}$a strictly increasing function of class $C^{1}$ such that: $\phi(0)=0$ and $\phi(t) \rightarrow+\infty$ as $t \rightarrow+\infty$. Assume that there exist $\sigma>0, \sigma^{\prime} \geq 0$ and $C>0$ such that:

$$
\begin{gathered}
\int_{S}^{\infty} E^{\sigma+1}(s) \phi^{\prime}(s) \mathrm{d} s \\
\leq C E^{\sigma+1}(S)+\frac{C}{(1+\phi(S))^{\sigma^{\prime}}} E^{\sigma}(0) E(S), \forall S \in \mathbb{R}^{+} .
\end{gathered}
$$

Then, there exists $C>0$ such that:

$$
E(t) \leq E(0) \frac{C}{(1+\phi(S))^{\frac{1+\sigma^{\prime}}{\sigma}}}, \forall t \in \mathbb{R}_{*}^{+}
$$

\section{Competing interests}

The authors declare that they have no competing interests.

\section{Authors' contributions}

All authors have contributed to all parts of the article. All authors read and approved the final manuscript. 


\section{References}

[1] F.D. Araruna and A.B. Maciel. Existence and boundary stabilization of the semilinear wave equation. Nonlinear Analysis: Theory, Methods and Applications, 67, 2007.

[2] MarceloM̃. Cavalcanti, ValÃ@ria N. Domingos Cavalcanti, and Irena Lasiecka. Well-posedness and optimal decay rates for the wave equation with nonlinear boundary damping source interaction. Journal of Differential Equations, 236, 2007.

[3] L.D̃iening, P. HÃåsto, P. Harjulehto, and M. Ru?zicka. Lebesgue and Sobolev Spaces with Variable Exponents, volume 2017. in: Springer Lecture Notes in Mathematics, Springer-Verlag, Berlin, 2011.

[4] L. Diening and M. Ru?zicka. Calderon Zygmund operators on generalized Lebesgue spaces $L^{p(x)}$ and problems related to fluid dynamics. Preprint Mathematische FakultÃat, Albert-Ludwigs-UniversitÃ at Freiburg, 120:197-220, (21/2002, 04.07.2002).

[5] X. L. Fan, J. Shen, and D. Zhao. Sobolev embedding theorems for spaces $W^{k, p(x)}(\Omega)$. J. Math. Anal. Appl., 262:749-760, 2001.

[6] Y. Fu. The existence of solutions for elliptic systems with nonuniform growth. Studia Math., 151:227-246, 2002.

[7] Ha Tae Gab. Blow-up for wave equation with weak boundary damping and source terms. Applied Mathematics Letters, $49,2015$.

[8] Tae Gab Ha. Blow-up for semilinear wave equation with boundary damping and source terms. Journal of Mathematical Analysis and Applications, 390, 2012.

[9] H.A.Levine and L.E.Payne. Non existence theorems for the heat equation with nonlinear boundary conditions and for the porousmedium equation back-ward in time. J. Differ. Equ, 16, 1974.

[10] O. KovÃ cik and J. RÃ $¡$ kosnik. On spaces $l^{p(x)}(\omega)$ and $w^{1, p(x)}(\omega)$. Czechoslovak Math. J., 41:592-618, 1991.

[11] Patrick Martinez. A new method to obtain decay rate estimates for dissipative systems. ESAIM Control Optimisation and Calculus of Variations, 4, 1999.

[12] M.M.Cavalcanti and V.N.Domingos Cavalcanti. Existence and asymptotic stability for evolution problems on manifolds with damping and sourceterms. J. Math. Anal. Appl, 291, 2004.

[13] M.M.Cavalcanti, V.N.Domingos Cavalcanti, and J.A.Soriano. Global solvability and asymptotic stability for the wave equation with nonlinearfeedback and source term on the boundary. Adv. Math. Sci. Appl, 16, 2006.

[14] M.M.Cavalcanti, V.N.Domingos Cavalcanti, and P.Martinez. Existence and decay rate estimates for the wave equation with non linear boundary damping and source term. J.Differ.Equ, 203, 2004.

[15] M.M.Cavalcanti, V.N.Domingos Cavalcanti, and P.Martinez. General decay rate estimates for viscoelastic dissipative systems. Nonlinear Anal, 68, 2008.

[16] Jong Yeoul Park and Tae Gab Ha. Energy decay for nondissipative distributed systems with boundary damping and source term. Nonlinear Analysis: Theory, Methods and Applications, 70, 2009.

[17] Jong Yeoul Park and Tae Gab Ha. Well-posedness and uniform decay rates for the klein?gordon equation with damping term and acoustic boundary conditions. J. Math. Phys, 50, 2009.

[18] Jong Yeoul Park, Tae Gab Ha, and Y. H. Kang. Energy decay rates for solutions of the wave equation with boundary damping and source term. J. Math. Phys, 61, 2010.

[19] Jong Yeoul Park, Tae Gab Ha, and Yong Han Kang. Energy decay rates for solutions of the wave equation with boundary damping

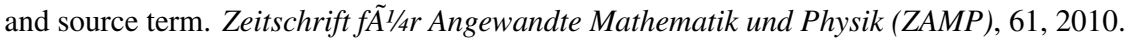

[20] Abita Rahmoune. Existence and asymptotic stability for the semilinear wave equation with variable-exponent nonlinearities. Journal of Mathematical Physics, 60, 122019.

[21] T.G.Ha. On viscoelastic wave equation with non linear boundary damping and source term. Commun. PureAppl. Anal, 9, 2010.

[22] Enzo Vitillaro. Global nonexistence theorems for a class of evolution equations with dissipation. Archive for Rational Mechanics and Analysis, 149, 1999.

[23] Enzo Vitillaro. Global existence for the wave equation with nonlinear boundary damping and source terms. Journal of Differential Equations, 186, 2002.

[24] V.Komornik and E.Zuazua. On viscoelastic wave equation with non linear boundary damping and source term. Commun. PureAppl. Anal, 69, 1990.

[25] Park Jong Yeoul and Ha Tae Gab. Existence and asymptotic stability for the semilinear wave equation with boundary damping and source term. Journal of Mathematical Physics, 49, 2008.

[26] E. Zuazua. Exponential decay for the semilinear wave equation with locally distributed damping. J. Math. Phys, 15, 1990. 